\title{
V-band Doppler backscattering diagnostic in the TCV Tokamak
}

P. Molina Cabrera, 1, a) S. Coda, L. Porte, N. Offeddu, P. Lavanchy, M. Silva, M. Toussaint, and the TCV team ${ }^{\text {b) }}$ Ecole Polytechnique Fédérale de Lausanne (EPFL), Swiss Plasma Center (SPC), CH-1015 Lausanne, Switzerland.

(Dated: 11 July 2018)

A variable configuration V-band heterodyne Doppler back-scattering diagnostic has been recently made operational in the Tokamak à Configuration Variable (TCV). This article describes the hardware set-up options, flexible quasi-optical launcher antenna, data-analysis techniques, and first data. The diagnostic uses a fast arbitrary waveform generator as the main oscillator and commercial vector network analyzer extension modules as the main mm-wave hardware. It allows sweepable single or multi-frequency operation. A flexible quasi-optical launcher antenna allows $3 \mathrm{D}$ poloidal $\left(10-58^{\circ}\right)$ and toroidal $\left(-180-180^{\circ}\right)$ steering of the beam with $0.2^{\circ}$ accuracy. A pair of fast $\mathrm{HE}_{11}$ miter-bend polarizers allow flexible coupling to either $\mathrm{O}$ or $\mathrm{X}$ mode and programmable polarization changes during the shot. These have been used to measure the magnetic-field pitch angle in the edge of the plasma by monitoring the backscattered signal power. Ray-tracing simulations reveal an available $\mathrm{k}_{\perp}$ range between 3 and $16 \mathrm{~cm}^{-1}$ with a resolution of $2-4 \mathrm{~cm}^{-1}$. Perpendicular rotation velocity estimates compare well against ExB plasma poloidal rotation estimates from charge exchange recombination spectroscopy.

\section{INTRODUCTION}

Plasma turbulence is actively studied in the Tokamak à Configuration Variable $(\mathrm{TCV})^{1}$. The present article presents the newest addition to the TCV turbulence diagnostic suite: a Doppler backscattering diagnostic (DBS). Prototype microwave reflectometers and DBS systems have been tested in TCV in the past, on loan from collaborators from the University of Stuttgart (Germany), Forschungszentrum Jülich (Germany), and the Laboratoire de Physique des Plasmas of Ecole Polytechnique Palaiseau (France $)^{2}$. However, the DBS system described in this article is the first effort to build a dedicated DBS diagnostic for TCV with long-term exploitation goals in mind.

DBS is an active diagnostic technique that allows the study of electron density turbulence via the scattering of a mm-wave beam launched at oblique incidence to a cut-off layer ${ }^{3},{ }^{4},{ }^{5}$. Launching a mmw beam at a macroscopic oblique angle to the cut-off surface allows the enhanced E-field amplitude near a plasma cut-off to localize the scattered power to where the refractive index reaches a minimum: the beam turning point. The scattering process relies on the presence of density fluctuations at this turning point and selects the probed fluctuation wavenumber according to the Bragg condition $\mathbf{k}_{f}$ $=-2 \mathbf{k}_{i}$ (where $\mathrm{f}$ refers to fluctuation and $\mathrm{i}$ to incident). If the fluctuations are moving with velocity $v$ with respect to the laboratory frame, the scattered wave will be Doppler shifted by $\Delta \omega=\mathbf{v} \cdot \mathbf{k}$. If the wave-vector of the incident beam is perpendicular to the main magnetic field at the turning point (which requires a small toroidal tilt of the antenna ${ }^{6}$ in tokamaks) the Doppler shift can be approximated to $\approx \mathrm{v}_{\perp} \mathrm{k}_{\perp}$. This perpendic-

\footnotetext{
a) pedro.molina@epfl.ch

b) See author list of S. Coda et al 2017 Nucl. Fusion 57102011
}

ular rotation velocity is the sum of the phase velocity of turbulence and a background plasma component, i. e.: $v_{\perp}=v_{\text {phase }}+v_{\mathrm{ExB}}$.

The present article begins by presenting the diagnostic hardware in section II and flexible quasi-optical antenna system in section III. Data analysis tools are presented in section IV. First results and conclusions follow in sections $\mathrm{V}$ and $\mathrm{VI}$, respectively.

\section{HARDWARE DESCRIPTION}

The DBS diagnostic presented here is capable of swept single and multi-channel operation in V-band through two interchangeable set-ups. The first, more conventional set-up, is shown in figure 1 . At the core sits a comercial off-the-shelf transceiver vector network analyzer (VNA) extension module from Virginia Diodes (VDI). To launch waves inside the $\mathrm{V}$ band, $\mathrm{mW}$-level sinusoidal signals in the range $8.33-12.5 \mathrm{GHz}$ are fed to the $\mathrm{RF}$ port of the TX/RX VDI module, wherein their frequency is multiplied by a factor of 6 using varactor multipliers. To generate these "low" frequency sinusoids, a fast arbitrary waveform generator (AWG - Keysight M8195A) with $25 \mathrm{GHz}$ analog bandwidth is used. This AWG is composed of a $65 \mathrm{GSa} / \mathrm{s}$ digital-to-analog converter coupled to a set of programmable digital filters. It produces about $4 \pm 2 \mathrm{dBm}$ inside the above-mentioned range. It can play up to $50 \mathrm{~ms}$ of arbitrary frequency sweeps from internal memory. Longer waveforms may also be played by engaging its sequencer capabilities. The output power in the V-band after the frequency multiplication ranges between 6 and 10dBm. A second AWG channel provides the local-oscillator ( $\mathrm{LO}$ ) input which is mixed with both outgoing and returning power to produce a heterodyne output in Reference and Measure ports in figure 1. An IF frequency of $1.88 \mathrm{GHz}$ is chosen to ensure minimal interference from spurious signals present in the toka- 
mak's electromagnetic environment and to minimize the $1 / \mathrm{f}$ noise contribution.

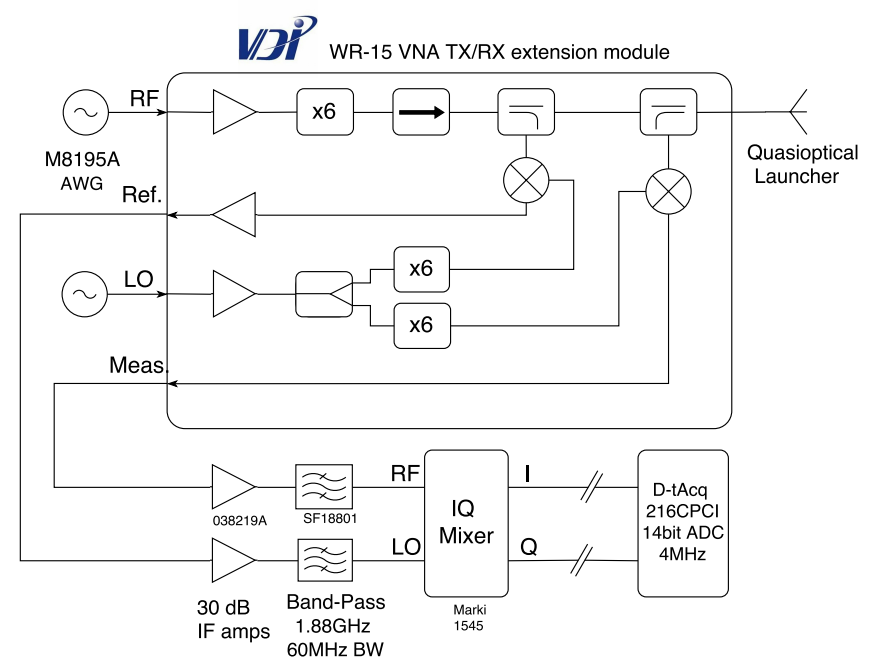

FIG. 1. Block diagram of the single-channel DBS setup.

In order to separate amplitude and phase in the heterodyne signal, hardware quadrature detection is used in the form of an I/Q mixer (Marki Microwave model 1545). Given that the power available in the reference and measure ports is often under $-25 \mathrm{dBm}$ and that the $\mathrm{I} / \mathrm{Q}$ mixer requires $+10 \mathrm{dBm}$ and $\sim 0 \mathrm{dBm}$ in the $\mathrm{LO}$ and $\mathrm{RF}$ ports respectively, broadband IF amplifiers $(0.01-3 \mathrm{GHz}$, 34dB gain, Fairview Microwave model SLNA-030-34-14) and narrow bandpass filters (BPF - $1.88 \mathrm{GHz}$ center frequency, $60 \mathrm{MHz}$ bandwidth, Fairview Microwave model SF18801) are used to condition power and reject spurious signals (coaxial cable pick-up and LO bleedthrough). I/Q amplitude deviation and quadrature phase deviation are under $0.2 \mathrm{~dB}$ and 3degrees, respectively. In order to protect the integrity of I/Q signals from the harsh electromagnetic environment in the TCV tokamak hall, the IF-amplifier, BPFs, and I/Q mixer hardware were installed next to the VDI Tx/Rx extension module. Also, the entire fixture was enclosed in a $2 \mathrm{~mm}$ thick aluminum shielding box (bottom right in figure 3). It is important to minimize the distance the Ref. and Meas. signals travel in coaxial cables, which although properly terminated were found sensitive to pick-up. This noise may be attributed to potentials generated due to the impedance mismatch (imbalance) between the coaxial cables' central conductor and shield in the presence of changing $\mathrm{E}$ fields. The I/Q signals are routed from the DBS box to the digitizer $(\sim 2 \mathrm{~m})$ through shielded twisted pair cables which are balanced and hence resilient against changing E fields. The I/Q signals are then sampled by a 14bit differential ADC (D-tAcq model 216CPCI) at 4MHz. The ADC contains an internal $5 \mathrm{MHz}$ anti-aliasing filter, which is used to reject LO bleed-through from the I/Q mixer and sample only Doppler shifts expected to be a few $\mathrm{MHz}$ at most. The ADC output is stored in the MDSPlus TCV database.
An alternative hardware set-up has been recently tested for the production and detection of multiple simultaneous frequencies. Leveraging the multi-tone capabilities of the fast AWG, a new method of multi-channel DBS has been developed. Conventional multi-channel DBS systems use a comb frequency generator (non-linear transmission lines), a frequency multiplier, and a fixed set of filters in a heterodyne receiver ${ }^{78}$. The newly proposed method consists of creating a double frequency spectrum with the AWG, feeding this signal into the varactor multipliers inside the VDI modules, and directly sampling the entire output spectrum with a fast oscilloscope as shown in figure 2. A receiver $(\mathrm{Rx})$ module is used for detection instead of the Tx/Rx module above because the Rx module features a larger IF bandwidth from $5 \mathrm{MHz}$ to $11 \mathrm{GHz}$. It is well known ${ }^{9}$ that the nonlinear response of frequency multipliers implies the generation of all frequency products of the form $n f 1+m f 2$ (where $\mathrm{n}$ and $\mathrm{m}$ are integers) to a double frequency input. Therefore, if a two-tone input composed of $\mathrm{f} 1$ and $\mathrm{f} 2$ is fed into the $\mathrm{x} 6$ VDI varactor multiplers, their output would consist of $6 \mathrm{f1} 1,6 \mathrm{f} 2$, and also (at least) 5 other intermodulation products in between, namely: $5 \mathrm{f} 1+1 \mathrm{f} 2,4 \mathrm{f} 1+2 \mathrm{f} 2,3 \mathrm{f} 1+3 \mathrm{f} 2,2 \mathrm{f} 1+4 \mathrm{f} 2$, $1 \mathrm{f} 1+5 \mathrm{f} 2$. The separation between these products corresponds to the initial frequency difference f1-f2. Seven frequencies can thus be sent into the plasma. The distance between the first two tones may be easily changed and is only limited by the maximum IF bandwidth of the receiver. A fast (Lecroy Wavemaster 813Zi-B) 8-bit oscilloscope with $13 \mathrm{GHz}$ analog bandwidth $(40 \mathrm{GSa} / \mathrm{s})$ is used to directly sample the IF output of the receiver mixer in figure 2. It is interesting to note that given the variable gain in the scope channels, no amplification was required to read spectra with SNR $\sim 20-30 \mathrm{~dB}$.

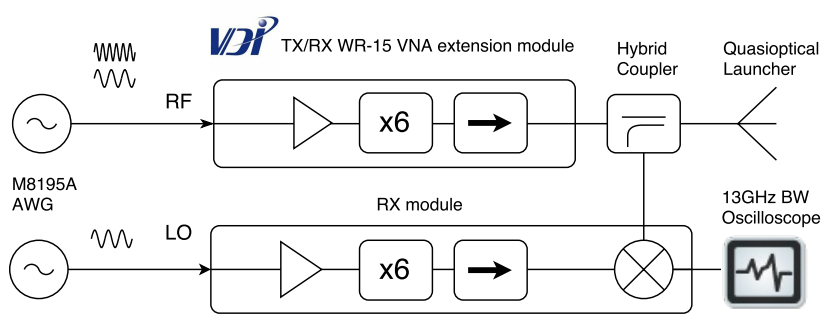

FIG. 2. Block diagram of the multi-channel DBS setup.

\section{THE LAUNCHER ANTENNA AND SIGNAL PATH}

The DBS diagnostic uses a quasi-optical launcher antenna inherited from TCV's second-harmonic electron cyclotron resonance heating (ECRH) launcher antennas, described in detail elsewhere ${ }^{10}$. This antenna allows 3D poloidal $\left(5-50^{\circ}\right)$ and toroidal $\left(-180-180^{\circ}\right)$ steering of the beam with $0.2^{\circ}$ accuracy. It can sweep through the entire poloidal range in $0.5 \mathrm{~s}$ and may be actively steered during a TCV shot. Figure 3 shows the path the signal follows 
from the output port of the transceiver module towards the launcher antenna.

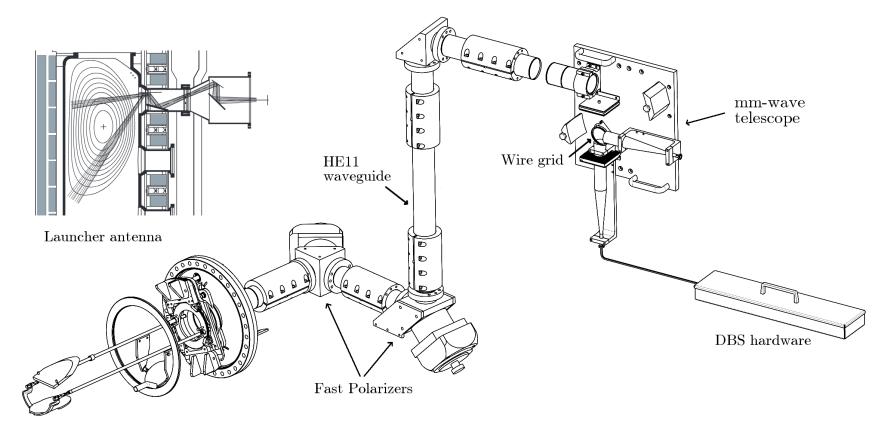

FIG. 3. Overview of waveguide path from DBS module to quasi-optical launcher antenna. A horizontal 4-meter $\mathrm{HE}_{11}$ waveguide section has been shortened for clarity and is shown as a gap above.

Firstly, the WR-15 rectangular waveguide output is tapered up to WR-19 (U band) and transformed from a fundamental $\mathrm{TE}_{10}$ mode into a free space fundamental TEM $_{00}$ mode (Gaussian beam) using a U-band gaussian horn antenna courtesy of Forschungszentrum Jülich. The Gaussian beam is then launched into a quasioptical telescope manufactured by Thomas Keating shown in the top right-hand side of figure 3 . The telescope allows two different perpendicular polarizations to be launched and/or received to and from the launcher antenna: a horizontal polarization coming from the bottom horn and a vertical polarization coming from the side horn. This is achieved using a wire-grid polarizer which reflects incident horizontal polarizations.

The next step couples the Gaussian beam to over 6.5 meters of circular oversized corrugated $\mathrm{HE}_{11}$ waveguide $(63.5 \mathrm{~mm}$ diameter). These waveguides feature good coupling to free-space gaussian modes, large bandwidth, little cross-polarization, and losses under $0.01 \%$ per meter ${ }^{11}$. Lastly, in order to control the in-plasma polarization, a pair of fast universal polarizers has been installed. These are waveguide miter bends with grooved mirrors that can be used to alter the polarization of the incident $\mathrm{HE}_{11}$ mode. Figure 3 shows two such polarizer miter bends in series (a polarization rotator and circular polarizer) that can produce any arbitrary output elliptical polarization when fed a linear polarization ${ }^{12}$. These polarizers had already been used in TCV for ECRH and oblique ECE applications; further technical details can be found elsewhere ${ }^{13}$. The ECPOL ${ }^{14}$ suite is used to optimize both polarizer angles for the best possible transmission of a particular linear polarization in the telescope to a target arbitrary elliptical polarization in the plasma. Although the $\mathrm{HE}_{11}$ waveguide and fast polarizers have been designed for $82.7 \mathrm{GHz}$, the DBS V-band frequencies can still be coupled to either $\mathrm{O}$ or $\mathrm{X}$ mode with $>90 \%$ power transmission. The output of the polarizers is directly fed to the quasi-optical launcher antenna. A quartz window acts as a vacuum seal at the interface between launcher and polarizers. The launcher antenna has been designed to maintain the input polarization at its steerable output but has been optimized for $82.7 \mathrm{GHz}$. First-order Gaussian beam parameter calculations indicate that $94.7 \%$ of the input power is transmitted through the 4 launcher mirrors at $50 \mathrm{GHz}$.

TCV is well-known for its high ECRH power density. Given that the damage threshold of the V-band VDI modules shown above in figure 1 is $0.1 \mathrm{~W}$, protecting the DBS hardware is paramount. A combined protection of $>86 \mathrm{~dB}$ exists for both $\mathrm{X} 2$ and $\mathrm{X} 3$ power in the horizontal telescope DBS beam path. Firstly, a QMC K1798 mesh low pass filter $(-3 \mathrm{~dB}$ point at $76 \mathrm{GHz})$ provides $26 \mathrm{~dB}$ rejection at $82.7 \mathrm{GHz}$ and over $30 \mathrm{~dB}$ for $118 \mathrm{GHz}$. It has been installed in the entrance of the horizontal horn in the telescope. Secondly, a U-band waveguide $82.7 \mathrm{GHz}$ notch filter (-60dB) built by Quinstar (QNF-083U60) has been placed directly after the horizontal (bottom) $\mathrm{U}$ band horn. Lastly, a V-band waveguide stripline low-pass filter from Pacific Microwave ( $-3 \mathrm{~dB}$ point at $86 \mathrm{GHz}$ ) provides $-60 \mathrm{~dB}$ rejection at $118 \mathrm{GHz}$ and is installed directly in front of the DBS module. No damage to DBS components has been encountered to-date.

\section{DATA ANALYSIS APPROACH}

\section{A. Ray tracing}

In order to correctly interpret the effective location and scattering wave number of the backscattered signal, it is imperative to model the trajectory of the microwave beam towards the turning point where the scattering takes place. Ray-tracing solves the wave equation within the framework of geometric optics making use of the WKB approximation. It evolves each ray independently. A 2D 3-point (where one ray represents the beam's centre and the other two describe the Gaussianbeam envelope $1 / \mathrm{e}$ amplitude points) ray-tracing routine called PrefGeom built in-house by C.A. de Meijere in 2013 using MATLAB ${ }^{\mathrm{TM}}$ has been expanded to plan and interpret DBS experiments. It firstly propagates a Gaussian beam through vacuum using Gaussianbeam complex q-parameter formalism ${ }^{15}$ from the mouth of the HE11 waveguide through the four mirrors of the launcher. Once the beam reaches the last mirror, it is launched into an empty TCV vessel as a first approximation to show the beam propagation in vacuum. Figure 4 shows the launcher geometry and the vacuum beam envelope in red. The cut-off layer is also shown in red (i.e. for $65 \mathrm{GHz}$ in fig 4) at discrete poloidal launching angles spanning the entire poloidal range of the launcher. The cold-plasma dispersion relation for $\mathrm{O} / \mathrm{X}$ mode waves is used to define this cut-off layer by evaluating the points where the refractive index $(\mathrm{N})$ goes from positive to negative. Computing $\mathrm{N}$ requires both density and magnetic field information. The magnetic equilibrium is obtained from the LIUQE ${ }^{16}$ reconstruction, which uses magnetic- 
sensor experimental data and is routinely performed after every shot. The density profile of the confined plasma is taken from TCV's Thomson scattering (TS) diagnostic, constrained by vertical far-infrared interferometer data. Given the large inhomogeneities inherent to the instantaneous nature of TS data, a cubic spline fit is performed on raw TS data.

The scrape-off-layer (SOL) density profile is approximated building on the latest findings on the subject ${ }^{17}$. The near SOL region $\left(\rho_{\psi} 1.0-1.025\right)$ profile is approximated with a exponential decay function of constant $\lambda_{n}=\left(\left|\nabla n_{e}\right| / n_{e}\right)^{-1} 1.5 \mathrm{~cm}$ and $3 \mathrm{~cm}$ in shots with core line average densities below and above $6 \cdot 10^{19} \mathrm{~m}^{-3}$, respectively. For the far SOL region $\left(\rho_{\psi}\right.$ 1.025-1.06), the exponential decay constant is $3 \mathrm{~cm}$ regardless of density. The SOL region is extended up to the $\rho_{\psi}$ that matches the outer-gap length calculated with the LIUQE reconstruction. The latter is defined as the distance between the outter most point (low-field side) of the last closed flux surface (LCFS) and the vessel wall. This outer-gap length effectively defines the SOL radial extent observed by the launcher. It is assumed that the contribution of the shadow-region's density profile to the refraction of the beam is negligible both because of its significantly lower density values and the short distances the beam passes through it. TCV's Psi-Toolbox suite ${ }^{18}$ is used to combine both density and magnetic profiles in $2 \mathrm{D}$ cylindrical R-Z geometry.

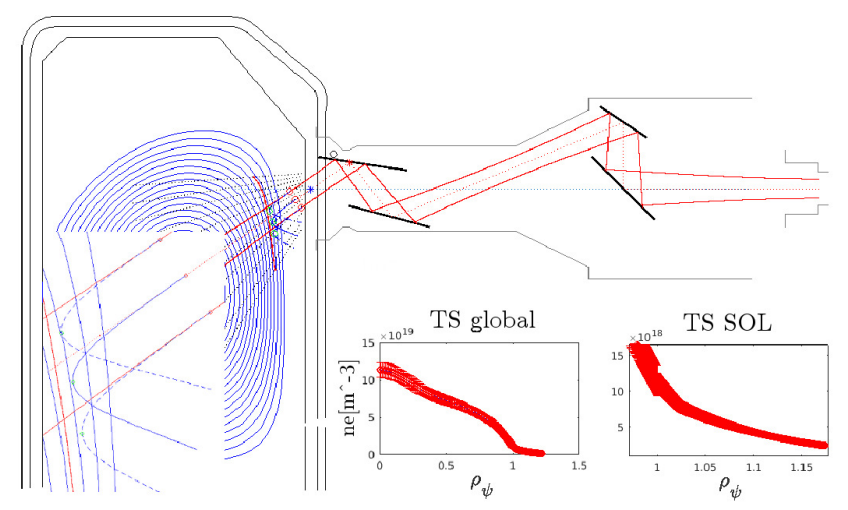

FIG. 4. PrefGeom 2D 3-point ray-tracing output for shot 59534 at $1.0 \mathrm{~s}, 65 \mathrm{GHz}$, and X-mode polarization. A closeup of the beam trajectory approaching the turning point is shown in the bottom left corner for clarity. Confined plasma and SOL density profiles are shown for reference in the bottom right corner.

Figure 4 shows the 3 -point ray-tracing starting positions at the edge of the SOL region. The ray-tracing equation used by PrefGeom is $\frac{d}{d s}(N \hat{s})=\nabla N^{19}$, where $\hat{s}$ is a unit vector whose direction is perpendicular to the wavefront. It is solved in 2D by MATLAB's ode45 solver. The initial direction of the central ray is defined by the launcher's poloidal angle. The top and bottom rays' locations and angles of divergence are defined by the local width and radius of curvature of an ideal Gaus- sian beam. At each point in each beam's trajectory, the refractive index is computed. When the refractive index reaches a minimum (shown with green circles in figure 4), the scattering wavenumber $\mathrm{k}_{\perp}$ is estimated using $\mathrm{k}_{\perp}=2$ $\mathrm{N}_{\min } \mathrm{k}_{o}{ }^{20}$. Since the ray-tracing takes place in a purely poloidal $2 \mathrm{D}$ geometry, the $\mathrm{N}_{\min }$ is not exactly $\mathrm{N}_{\perp}$, but the difference in tokamaks is expected to be small. It has been empirically shown by Conway et al. ${ }^{21}$ that 3 -point ray-tracing offers an estimate of the fundamental $\Delta \mathrm{k}_{\perp}$ wavenumber and $\Delta \rho_{\psi}$ localization resolutions. Figure 5 shows a k-accessibility map for a negative triangularity Lmode TCV plasma, which provides near slab-like plasma cutoff layers.

Figure 5 also shows the effects of different SOL profiles in determining the turning point localization and wavenumber. The so-called exp profile has been introduced above, and it is used by default. The lin profile refers to an unrealistic SOL profile that decreases linearly from $\rho=1$ to zero at $\rho=1.125$ regardless of the plasma conditions. For both of these, the ray-tracing is began before entering the SOL (in the so-called shadow ${ }^{17}$ region). The vac profile refers to treating the SOL as vacuum, propagating the beam using complex q parameters until reaching the LCFS, and starting the ray-tracing calculation just before $(0.5 \mathrm{~mm})$ the interface.

Comparing results between linear and exponential SOL density profiles shows differences under $1 \mathrm{~cm}^{-1}(\sim 10 \%)$ inside $\rho_{\psi}<1$. Uncertainties in $\mathrm{k}_{\perp}$ due to the finite width of the probing beam (from 3-point ray tracing) are usually found inside $\Delta \mathrm{k}_{\perp} 2-4 \mathrm{~cm}^{-1}$. Thus, the differences between both SOL profiles in $\mathrm{k}_{\perp}$ estimates lie well inside error bars. Localization differences between the two in $\rho_{\psi}$ are much smaller and are found an order of magnitude under $\rho_{\psi}$ uncertainties from 3-point ray tracing $\sim 0.005-0.015$. However, the differences are much more significant when comparing both SOL profiles and the vacuum case. The differences in $\mathrm{k}_{\perp}$ estimates of turning points $\rho_{\psi}>0.96$ are comparable to the 3 -point $\Delta \mathrm{k}_{\perp}$, constantly understimating the scattering wave-vector. For $\mathrm{k}_{\perp}>8 \mathrm{~cm}^{-1}$, the vacuum estimates are significantly underestimated regardless of $\rho_{\psi}$. Turning-point localization discrepancies between the two SOL profiles and vacuum are not as pronounced, but they may become relevant (outside error bars) for $\mathrm{k}_{\perp}>10 \mathrm{~cm}^{-1}$ and $\rho_{\psi}<0.95$. It can be concluded from this figure tlvat if the $\mathrm{k}_{\perp}$ measured is above $8 \mathrm{~cm}^{-1}$ and/or has turning points above 0.95 , the beam's refraction inside the SOL plays an important role in the accuracy of wavenumber estimates.

\section{B. Power spectral density and Doppler shift estimates}

DBS can infer the poloidal rotation of electron turbulence by measuring the Doppler shift (fD) of scattered signals. In order to extract this $\mathrm{fD}$ from the phasor defined by the I/Q signals, a two-sided Power Spectral Density (PSD) estimate is obtained using the Welch method ${ }^{22}$. Data acquired for $1 \mathrm{~ms}$ at $4 \mathrm{MHz}$ is split into 8 segments 


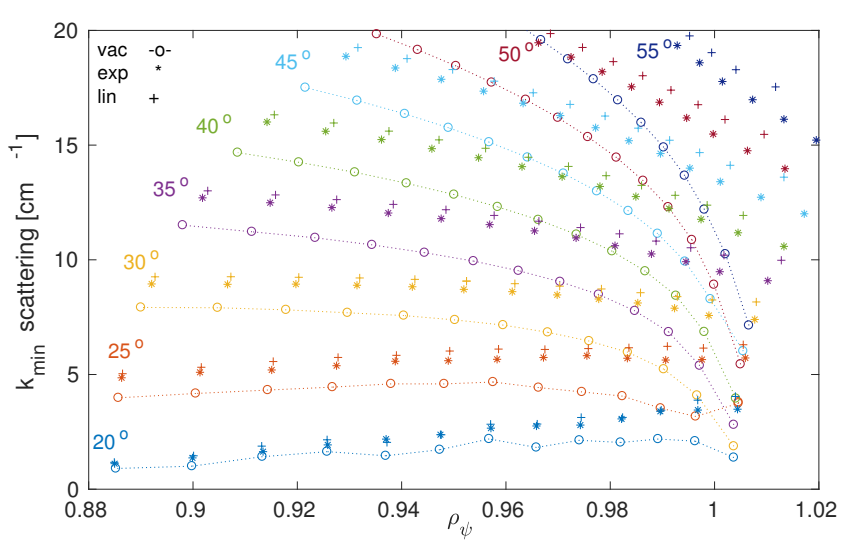

FIG. 5. $\mathrm{k}_{\perp}$ accessibility map for shot 59534 at 1.0 s varying the launcher's poloidal angle between 56 and 20 degrees. The turning-point location depends on frequency going from 50 to $74 \mathrm{GHz}$ in steps of $2 \mathrm{GHz}$. $\mathrm{k}_{\perp}$ and $\rho_{\psi}$ uncertainties are found inside $2-4 \mathrm{~cm}^{-1}$ and $0.005-0.015$, respectively, and are omitted above for clarity. The $\mathrm{k}_{\perp}$ axis extends up to $20 \mathrm{~m}^{-1}$ for illustrative purposes only. Useful signals are usually only measured up to $\sim 16 \mathrm{~m}^{-1}$.

and FFTs of each segment are averaged using a Hanning window to avoid spectral leakage. Each segment features $50 \%$ overlapping. The error bars are calculated with $\sqrt{11 /(18 N)}$ where $\mathrm{N}$ is the number of segments used in the Welch average. Figure 6 shows a sample PSD snap of I/Q data acquired during shot 59555 at about $0.7 \mathrm{~s}$. Smoothing is firstly applied to the Welch PSD output in $\mathrm{dB}(\log )$. The rigid LOESS method ${ }^{23}$, a generalization of the robust locally weighted regression method, has been chosen through MATLAB's built-in smooth function. The next step is to determine the peak location in frequency from the maximum of the smooth PSD spectra, which is done by fitting a scaled skewed normal (SN) distribution function ${ }^{24}$.

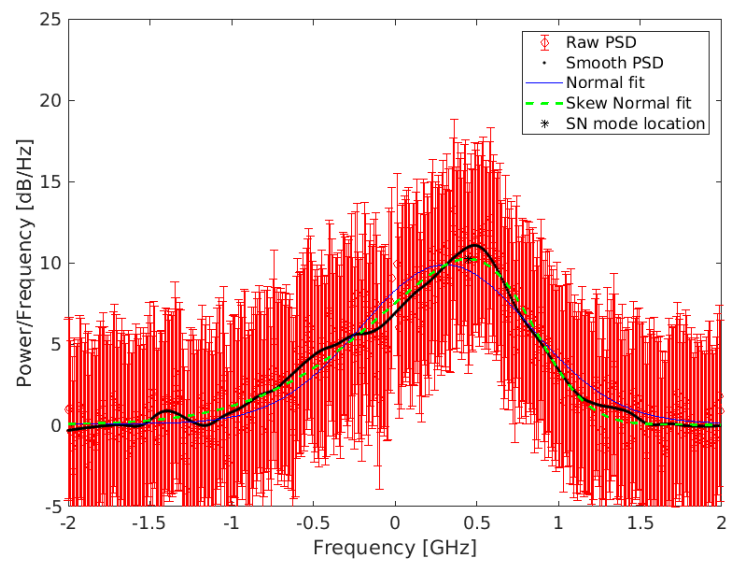

FIG. 6. PSD estimate and fD fits. Shot 59555 at $0.7 \mathrm{~s} . \mathrm{k}_{\perp}=$ $5.4 \pm 4 \mathrm{~cm}^{-1} \cdot \rho_{\psi}=0.967 \pm 0.002$.

$$
\mathrm{PDF}=\frac{2}{\omega \sqrt{2 \pi}} e^{-\frac{(x-\xi)^{2}}{2 \omega^{2}}} \int_{-\infty}^{\alpha\left(\frac{x-\xi}{\omega}\right)} e^{-\frac{t^{2}}{2}} d t
$$

where $\xi$ is the location parameter, $\omega$ is the scale parameter, and $\alpha$ is the slant parameter that allows asymmetry in the PDF. The normal distribution function is recovered when $\alpha$ is equal to zero. The integral in equation 1 above is estimated using MATLAB's normcdf function. Figure 6 shows both a generic Gaussian and the $\mathrm{SN}$ fit and how the SN fit better estimates the peak of the Doppler shift. In order to estimate Doppler shift, the location of the maximum in the SN fit is found using accurate analytic estimates of the SN distribution's mode (available here ${ }^{24}$ ). In order to estimate the uncertainty in the mode, the standard error propagation formula is applied to the mode formula. The uncertainty in each fit parameter is obtained from the fit's $95 \%$ confidence interval estimates.

\section{Uncertainty estimates}

The turbulence perpendicular rotation velocity is estimated by $v_{\perp}=2 \pi \mathrm{fD} / k_{\perp} . \mathrm{k}_{\perp}$ and $\mathrm{fD}$ uncertainties are found with 3-point ray-tracing and through fit 95\% confidence intervals as explained above. In addition to these, in TCV, the TS density profile features error bars of $10 \%$. Thus, in order to estimate the effect of such error bars on the scattering wavenumber and the localization, the density profile is artificially changed to 110 and $90 \%$ and TSdriven $\Delta \mathrm{k}$ and $\Delta \rho_{\psi}$ are calculated. In order to keep the TS uncertainties separate from the inherent uncertainty of the DBS technique, average TS-driven error bars have been included in figures 7 and 9 . These show that the TS uncertainty has a strong effect in the $\rho_{\psi}$ localization and not in the $\mathrm{k}_{\perp}$. Errors introduced by the position of the launcher's poloidal angle ( $0.2^{\circ}$ resolution) are found to be neglegible compared to these.

\section{RESULTS AND DISCUSSION}

\section{A. DBS-CXRS comparison}

Assuming typical magnetic field values in TCV, the V band DBS system presented here may sample densities in the range 3 to 7 (O-mode) and 0.8 to 4 (X-mode) $\times$ $10^{19} \mathrm{~m}^{-3}$ which cover a large range of TCV's density operational space. The average SNR of the DBS signal is usually $20 \mathrm{~dB}$ and may fluctuate between 10 and $40 \mathrm{~dB}$ depending on shot conditions and $\mathrm{k}_{\perp}$. Doppler shifts are often found under $0.75 \mathrm{MHz}$ in L-mode and may reach $1.5 \mathrm{MHz}$ in $\mathrm{H}$-mode. The full-width at half max of the spectrum is usually $0.5-1 \mathrm{MHz}$. Doppler shift uncertainties are usually found in the $50-200 \mathrm{kHz}$ range. Single-frequency DBS turbulence $\mathrm{v}_{\perp}$ rotation estimates 
are compared with ExB poloidal rotation measurements from the charge-exchange recombination spectroscopy (CXRS) diagnostic in figure 7. Positive velocity in this figure refers to the electron diamagnetic drift direction, vertically upwards in figure 4 . CXRS measures the poloidal rotation of carbon impurities and invokes radial force balance to infer the bulk-plasma ExB contribution. The new high-resolution edge CXRS spectrometer ${ }^{25}$ was used in this comparison. The L-mode discharge 59555 shows relatively good agreement. In the ohmic H-mode shot 59558, agreement is still good but lies almost outside error-bars for the outermost two points. Results may be affected in $\rho_{\psi}$ by the instantaneous nature of the TS profiles taken at $1.417 \mathrm{~s}$. Furthermore, the $20 \mathrm{~ms}$ instantaneous stair-case sweeping of single-channel DBS frequencies samples the plasma differently than the simultaneous multi-channel $35 \mathrm{~ms}$ averages in CXRS in the time domain.

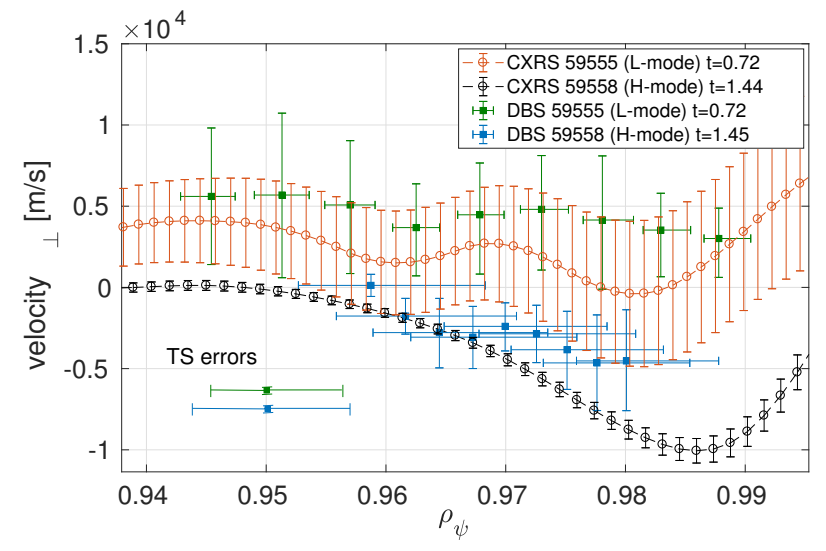

FIG. 7. Comparing DBS perpendicular turbulence velocity estimates with CXRS $v_{\operatorname{ExB}}$ estimates in L-mode $\left(59555, \mathrm{k}_{\perp}\right.$ $\left.5-5.8 \mathrm{~cm}^{-1}\right)$ and $\mathrm{H}$-mode $\left(59558, \mathrm{k}_{\perp} 6.5-7.5 \mathrm{~cm}^{-1}\right)$ discharges. The average TS contribution to $\Delta \rho_{\psi}$ and $\Delta \mathrm{v}$ are shown decoupled from the inherent DBS uncertainties for illustrative purposes.

\section{B. Multi-channel DBS}

Figure 8 shows the full IF PSD spectra recorded with the scope in figure 2 at $40 \mathrm{Gsa} / \mathrm{s}$ for $0.4 \mathrm{~ms}$ at time $0.7 \mathrm{~s}$ in shot 59551 . The two-tone input comprised $\mathrm{f} 1=8.33 \mathrm{GHz}$ and $\mathrm{f} 2=9.33 \mathrm{GHz}$, which launched 50 and $56 \mathrm{GHz}$ as $\mathrm{x} 6$ multiplication products. The LO input was $8.325 \mathrm{GHz}$, which resulted in an IF frequency of $50 \mathrm{MHz}$ for $\mathrm{f} 1$ and $6.05 \mathrm{GHz}$ for $\mathrm{f} 2$ as can be seen in figure 8 . The analysis consists simply of focusing around each relevant frequency term and applying the fitting routines and raytracing outlined above. It is important to note the presence of other strong frequency terms in the spectra in figure 8 . These were not the focus of the analysis but are likely useful intermodulation terms above and below $6 \mathrm{f} 2$ and $6 \mathrm{f} 1$, respectively.

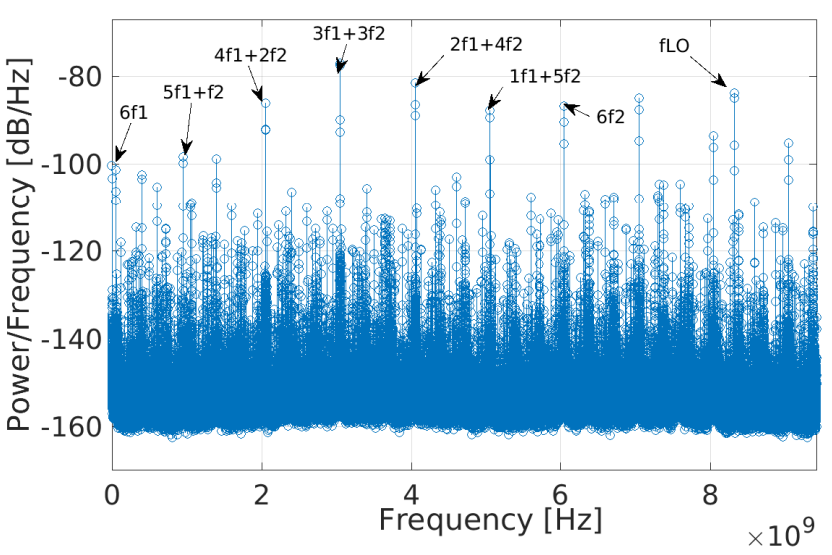

FIG. 8. Raw IF PSD spectra recorded by the fast scope showing the main multiplication and intermodulation terms used in the analysis of Doppler shifts in shot 59551

This new approach to multi-channel DBS has both advantages and disadvantages. Firstly, the non-linear-transmission-lines (NLTL) of comb-frequency generators $^{78}$ are not required. Furthermore, the fixed filter banks in the receiver are all substituted by the fast scope which records the Doppler shifts of each frequency simultaneously. Perhaps the strongest asset is that the multi-frequency output can change arbitrarily to best fit specific plasma conditions, conceivably inside the shot. Figure 9 shows the velocity estimates of three identical shots at time $0.7 \mathrm{~s}$ where the frequency spacing between the inputs was varied between $0.5,1$, and $2 \mathrm{GHz}$ resulting in seven output frequencies between 50-53, 5056 , and $50-62 \mathrm{GHz}$, respectively. Figure 9 shows that the different estimates agree with each other and with the frequency-swept DBS using conventional I/Q detection. A serious disadvantage of the current approach is that the plasma could be sampled only for as long as $\sim 2 \mathrm{~ms}$ given the limited scope memory $(32 \mathrm{Mb})$. Multiple triggering may allow sampling of up to $8 \times 250 \mu$ s instants during a shot, which remains impractical for routine measurements. This limitation maybe overcome by dataacquisition solutions which feature memories of on the 100s of gigabytes (Guzik ADP7000 for example) which could allow sampling of the entire TCV discharge with varying frequency spacing inside the shot. Another limitation is that the power dependance on frequency follows a near-parabolic curve. This could be a limitation for studies of relative fluctuation levels and k-spectrum, but it does not affect the determination of Doppler shifts. Either an in-vessel power calibration and/or E-H tuners ${ }^{7}$ could be used in future to allow such studies.

\section{In-shot polarization rotation to infer B-field line pitch}

The fast HE11 polarizer miter bends presented in section III have been used to change the inclination angle 


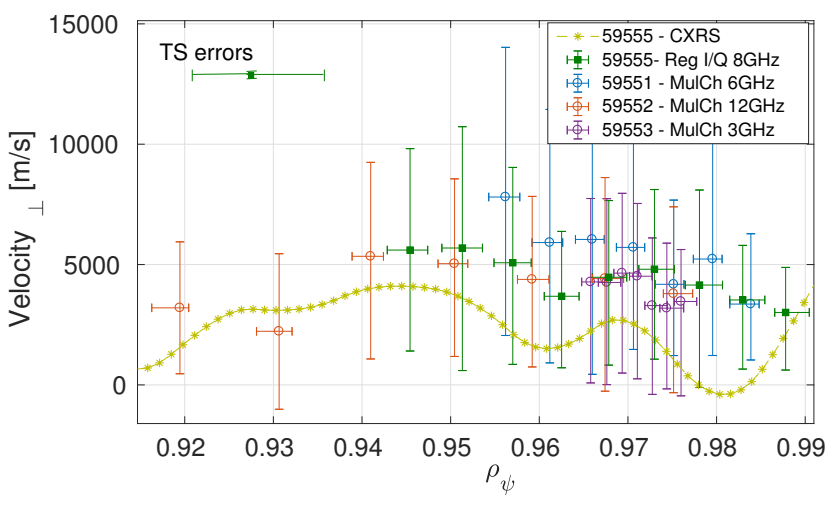

FIG. 9. AWG-driven multi-channel DBS validation during similar shot conditions $59551-55$ at $0.7 \mathrm{~s} . \mathrm{k}_{\perp} 4.5-6.0 \mathrm{~cm}^{-1}$. CXRS traces as shown in figure 7 are included without errorbars for clarity.

of the polarization ellipse $\alpha$ while keeping a constant elliptical polarization angle $\beta$ (formally defined here ${ }^{26}$ ) in search of an independant measurement of the magnetic field-line pitch. The best coupling (ideal $\alpha$ and $\beta$ ) to either $\mathrm{X}$ or $\mathrm{O}$ mode can be calculated by matching the launching beam wavevector and polarization to the relevant mode in the plasma's LCFS based on the magnetic field reconstruction. If the plasma conditions are stable, the coupling of the launched wave to either $\mathrm{X}$ or $\mathrm{O}$ mode at the plasma LCFS could be made to change by varying $\alpha$ while keeping $\beta$ constant. If both the effective $\alpha$ and the power of the DBS signal during the shot are examined, a measurement of the magnetic field pitch angle at the edge of the plasma is possible.

The $\alpha$ angle is measured with respect to the horizontal lab-frame ${ }^{14}$, to which the edge B-field line usually has an inclination between 5-15 degrees. Ideally, the $\mathrm{X}$ or Omode cut-off to be sampled would be located in close proximity to the LCFS. This would minimize power variations caused by changing plasma conditions between the LCFS and the cut-off location, which may induce spurious power variations. Also, preferably, the cut-off of the opposite mode to be scanned should be found further separated in $\rho_{\psi}$ from the LCFS and/or scatter at a larger $\mathrm{k}_{\perp}$, so that backscattered power from the main mode (cut-off closer to the LCFS) is significantly larger and a clear coupling maximum can be found.

Figure 10 shows time traces of both $\alpha$ and $\beta$ angles during shot 59679. The encoder output of both linear and elliptical miter bend polarizer angles is mapped onto $\alpha$ and $\beta$ maps over the shot time. Figure 10 shows that the effective $\alpha$ changes between +20 and -40 degrees while $\beta$ can be made to remain between -5 and -6 degrees , aiming for an ideal -5.36 degrees for X-mode best coupling at a chosen toroidal angle of 0 degrees. The best coupling to the X-mode wave into the main plasma (and then towards the scattering point expected at $\left.\rho_{\psi} 0.97 \pm 0.01\right)$ should occur when the probing beam's $\alpha$ hits a perfect $90^{\circ}$ to the LCFS B-field line.

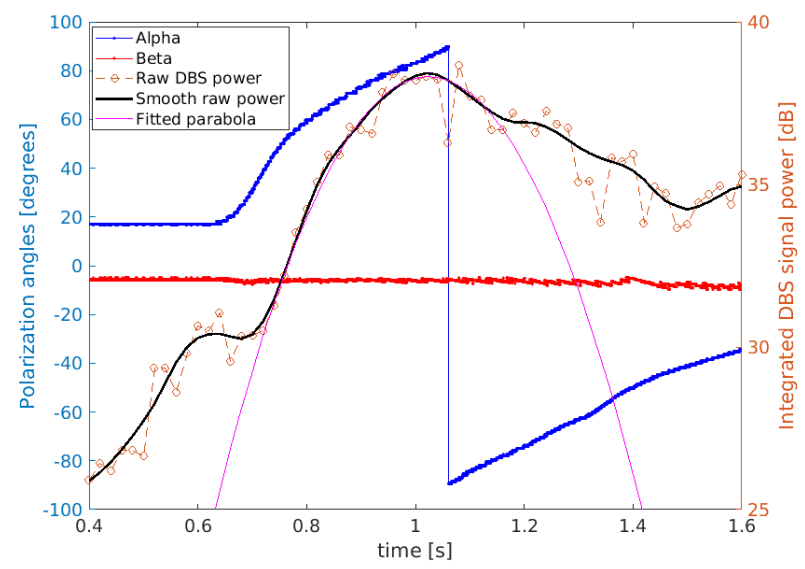

FIG. 10. DBS signal power evolution over changing polarization axis inclination $(\alpha)$ angle: i.e. coupling into $\mathrm{X} / \mathrm{O}-$ mode. Shot 59679. Output frequency was $60 \mathrm{GHz}$. Poloidal and toroidal angles are 18 and 0 degrees respectively. X-mode turning point at $\rho=0.97 \pm 0.01$ with $\mathrm{k}_{\perp}=8.2 \pm 2 \mathrm{~cm}^{-1}$. Omode turning point expected at $\rho=0.89 \pm 0.01$ with $\mathrm{k}_{\perp}=10.5$ $\pm 2 \mathrm{~cm}^{-1}$

The DBS signal power is estimated by integrating the area under the SN fits to the smooth PSD spectra described in section IV. After smoothing the raw DBS power points, a parabola $\left(a-\left((b *(x-c))^{2}\right)\right.$ is fit to the points around the peak power to determine the time of the peak DBS signal power. This peak is found at time $1.02 \pm 0.01$ where the alpha angle was $84.5 \pm 3$. The error in the time is taken from the $95 \%$ confidence interval in the fit for the constant $c$ above. The adjusted $\mathrm{R}^{2}$ is about 0.98 . The best $\mathrm{X}$-mode coupling $\alpha$ estimate agrees within uncertainty with the LIUQE suggestion of $81.7 \pm$ $0.4^{\circ}$. The LIUQE field pitch uncertainty was computed assuming that the largest contribution to the error in the field-line pitch at the LCFS is the reconstruction of the plasma position. Position errors obtained from covariance error analysis in the reconstruction of the magnetic axis position ${ }^{27}$ are mutiplied by a factor of two to obtain $0.6 \mathrm{~cm}$ in the vertical and $0.3 \mathrm{~cm}$ in the radial dimensions as an upper boundary. The error in the poloidal field is found by multiplying the local gradient of the poloidal field at the scattering location by the amplitude of the displacement chosen above. The error propagation formula is then used to estimate the error in the field pitch angle assuming no error in the toroidal field.

It can be concluded that the current experimental uncertainty must decrease by at least an order of magnitude to make this method practically relevant. In order to realize the polarization changes shown in figure 10, the elliptical and linear miter-bend polarizer stages moved by $128^{\circ}$ and $62^{\circ}$, respectively, in about one second. Considering the peak-torque of $12 \mathrm{Nm}$ of the RD-150 stepper motor drivers, the units could have theoretically achieved such trajectory in less than $100 \mathrm{~ms}$, which would have 
contributed to a 10x narrower parabola and less uncertainty in the peak coupling. Unfortunately, permanent increased mechanical torque in the elliptical unit due to friction between the mirror and the miter-bend casing prevented such faster trajectories to be realized due to driver overheating. A repair is foreseen by late November 2018. Another potential contributor to a smaller uncertainty could be the shortening of the $\alpha$ range used to find the maximum coupling. Figure 10 fits the DBS power only inside $\alpha \pm 70^{\circ}$ ( $36^{\circ}$ for the elliptical and $12^{\circ}$ for the linear units), which could have been theoretically achieved in about 50ms. Additionally, both motors could be programmed to turn continously at carefully synchronized rates that perform an $\alpha$ scan at constant $\beta$ during predetermined sections of a revolution. Such approach would avoid the (de)acceleration stages where the largest torque in the trajectory is found.

Lastly, a stronger DBS signal could have also contributed to less uncertainty. A larger SNR could be achieved by introducing a non-zero toroidal angle and aligning the wave-vector of the beam as perpendicular as possible to the field line based on the LIUQE field pitch estimates. The toroidal angle was chosen at $0^{\circ}$ in this discharge because the PrefGeom ray-tracing routine is currently 2D and cannot model a beam with a non-zero toroidal angle. Further experiments are planned once the elliptical fast polarizer unit is repaired.

\section{SUMMARY AND CONCLUSIONS}

The present article reports on the newly-installed Vband heterodyne DBS diagnostic in the TCV tokamak. The diagnostic can be uniquely configured for both sweepable single and multi-channel operation and is coupled to a flexible steerable quasi-optical launcher with in-shot adjustable polarization stages. It may reach densities between 0.8-7 $\times 10^{19} \mathrm{~m}^{-3}$. The accessible $\mathrm{k}_{\perp}$ range has been determined as 3-16 $\pm 2-4 \mathrm{~cm}^{-1}$ using $2 \mathrm{D}$ 3-point ray-tracing. The first results show DBS electron turbulence perpendicular rotation velocies in agreement with ExB poloidal rotation estimates from the CXRS diagnostic. Innovative experiments demonstrate a new approach to generating and detecting multiple simultaneous frequencies using a digital AWG and direct sampling. Furthermore, the magnetic-field line pitch angle at the edge was estimated by rotating the polarization axis orientation of the probing beam while maintaining a constant ellipticity, demonstrating unique $\mathrm{O} / \mathrm{X}$-mode coupling capabilities during the shot made possible by fast HE11 polarizers.

\section{ACKNOWLEDGMENTS}

Stefano Alberti and Timothy Goodman are thankfully acknowledged for helpful discussions and for facilitating access to low-power mmw hardware at SPC. The au- thors are also grateful to Claudio Marini for collecting the CXRS data used for comparison. Jeffrey Hesler from Virginia Diodes is thankfully acknowledged for prompt feedback and helpful discussions regarding the performance parameters of the VNA extension modules. Cedric Tsui is gratefully acknowledged for helpful discussions regarding SOL density profiles. This work is partially supported by Requip grant (No. 206021-150707) of the Swiss National Science Foundation and by the Helmholtz Virtual Institute on Plasma Dynamical Processes and Turbulence Studies using Advanced Microwave Diagnostics. This work has been carried out within the framework of the EUROfusion Consortium and has received funding from the Euratom research and training programme 2014-2018 under grant agreement No 633053. The views and opinions expressed herein do not necessarily reflect those of the European Commission.

\footnotetext{
${ }^{1}$ S. Coda, et.al. Overview of the TCV Tokamak Program : Scientific Progress and Facility Upgrades. Nuclear Fusion, 57(102011):12, 2017.

${ }^{2}$ C.A. de Meijere, S. Coda, Z. Huang, L. Vermare, T. Vernay1, V. Vuille, S. Brunner, J. Dominski, P. Hennequin, A. KrämerFlecken, G. Merlo, L. Porte, and L. Villard. Complete multifield characterization of the geodesic acoustic mode in the TCV tokamak. Plasma Phys. Contr. Fusion, 56(7):072001, 2014.

${ }^{3}$ G D Conway, J Schirmer, S Klenge, W Suttrop, E Holzhauer, and the Asdex Upgrade Team. Plasma rotation profile measurements using Doppler reflectometry. Plasma Phys. Contr. Fusion, 46(6):951-970, jun 2004.

${ }^{4}$ P. Hennequin, C. Honoré, A. Truc, A. Quéméneur, N. Lemoine, J. M. Chareau, and R. Sabot. Doppier backscattering system for measuring fluctuations and their perpendicular velocity on Tore Supra. Rev. Sci. Instrum., 75(10 II):3881-3883, 2004.

${ }^{5} \mathrm{~J}$ C Hillesheim, $\mathrm{W}$ a Peebles, $\mathrm{T}$ L Rhodes, L Schmitz, $\mathrm{T}$ a Carter, P-a Gourdain, and G Wang. A multichannel, frequencymodulated, tunable Doppler backscattering and reflectometry system. Rev. Sci. Instrum., 80(8):083507, 2009.

${ }^{6} \mathrm{C}$ Honoré, P Hennequin, A Truc, and A Quéméneur. Quasioptical Gaussian beam tracing to evaluate Doppler backscattering conditions. Nuclear Fusion, 46(9):S809-S815, 2006.

${ }^{7}$ W. A. Peebles, T. L. Rhodes, J. C. Hillesheim, L. Zeng, and C. Wannberg. A novel, multichannel, comb-frequency Doppler backscatter system. Review of Scientific Instruments, 81(10), 2010.

${ }^{8}$ R. Soga, T. Tokuzawa, K. Y. Watanabe, K. Tanaka, I. Yamada, S. Inagaki, and N. Kasuya. Developments of frequency comb microwave reflectometer for the interchange mode observations in LHD plasma. Journal of Instrumentation, 11(2), 2016.

${ }^{9}$ Pozar, D. Microwave Engineering. Addison-Wesley Publishing Company Inc., Reading, Massachusets, 1993.

${ }^{10}$ T. P. Goodman, V. S. Udintsev, I. Klimanov, A. Mueck, O. Sauter, and C. Schlatter. First measurements of oblique ECE with a real-time movable line of sight on TCV. Fusion Science and Technology, 53(1):196-207, 2008.

${ }^{11} \mathrm{~J}$. L. Doane. Mode converters for generating the HE 11 ( gaussian-like ) mode from TE 01 in a circular waveguide. Technical Report 1927, Princeton University, Princeton, New Yersey, 1982.

${ }^{12}$ J. Doane, H. Grunloh, W. Martin, and W. Wu. Polarizer miter bends for high-power microwave transmission: Ohmic loss and cooling. Fusion Engineering and Design, 102:99-107, 2016.

${ }^{13}$ M. Silva, T. Goodman, F. Felici, and L. Porte. Fast polarizers installation for ECRH and ECE in TCV. Fusion Engineering and Design, 86(6-8):1256-1259, 2011.

${ }^{14}$ F. Felici ECPOL : equations and Matlab tools for EC wave reflection and polarization calculations. Technical report, LRP
} 
01/2012, Swiss Plasma Centre, EPFL, Lausanne, Switzerland, 2012.

${ }^{15} \mathrm{H}$ J Hartfuss and Thomas Geist. Fusion Plasma Diagnostics with mm-Waves - An Introduction. Wiley-VCH - Physics Textbooks, 2013.

${ }^{16} \mathrm{~F}$ Hofmann and $\mathrm{G}$ Tonetti. Tokamak equilibrium reconstruction using Faraday rotation measurements. Nucl. Fusion, 28, 1988.

${ }^{17}$ N. Vianello, C. Tsui, C. Theiler, S. Allan, J. Boedo, B. Labit, H. Reimerdes, K. Verhaegh, W. A.J. Vijvers, N. Walkden, S. Costea, J. Kovacic, C. Ionita, V. Naulin, A. H. Nielsen, J. Juul Rasmussen, B. Schneider, R. Schrittwieser, M. Spolaore, D. Carralero, J. Madsen, B. Lipschultz, and F. Militello. Modification of SOL profiles and fluctuations with line-average density and divertor flux expansion in TCV. Nuclear Fusion, 57(11), 2017.

${ }^{18} \mathrm{~J}$. M. Moret. A software package to manipulate space dependencies and geometry in magnetic confinement fusion. Review of Scientific Instruments, 76(7), 2005.

${ }^{19}$ Elmore, W. and Heald, M. Physics of Waves. McGraw-Hill Inc., New York, NY, first edit edition, 1969.

${ }^{20}$ G. D. Conway, E. Poli, T. Happel, and the ASDEX Upgrade Team. Interaction of Mean and Oscillating Plasma Flows Across Confinement Mode Transitions. Plasma and Fusion Research,
5:S2005-S2005, 2010.

${ }^{21}$ G. D. Conway, C. Lechte, A. Fochi Assessment of Doppler reflectometry accuracy using full-wave codes with comparison to beam-tracing and analytic expressions. In 12th International Reflectometry Workshop, volume 2015, pages 1-14, 2015.

${ }^{22}$ P. D. Welch. The Use of Fast Fourier Transform for the Estimation of Power Spectra: A Method Based on Time Averaging Over Short, Modified Periodograms. IEEE Transactions on Audio and Electroacoustics, 15(2):70-73, 1967.

${ }^{23}$ W.S. Cleveland. Robust Locally Weighted Regression and Smoothing Scatterplots. Journal of the American Statistical Association, 74(368), 1979.

${ }^{24}$ Azzalini, A. The Skew-Normal and Related Families. Cambridge University Press, first edition, 2013.

${ }^{25}$ Marini, C. Poloidal CX visible light plasma rotation diagnostics in TCV. Phd thesis, Ecole Polytechnique Federale de Lausanne, 2017.

${ }^{26}$ Max Born and Emil Wolf. Principles of Optics. Cambridge University Press, Cambridge, UK, 7th edition, 1999.

${ }^{27}$ Dellaferrera, G. Covariance analysis and error measurement characterization of Tokamak equilibrium reconstruction. Technical report, Swiss Plasma Centre. Ecole Polytechnique Federale de Lausanne, Lausanne, Switzerland, 2018. 